\title{
BIO_17 - Screening of New Protease Inhibitors with Application in the Treatment of HCV Infections non-Responsible to the Direct-Action Antivirals
}

Rômulo José Soares Bezerra ${ }^{1 *}$; Joana Miranda Pereira ${ }^{1}$; Priscila Conrado Guerra Nunes ${ }^{1}$; José Henrique da Silva Pilotto ${ }^{1}$; Fernando de Carvalho da Silva ${ }^{2}$; Jaqueline Mendes de Oliveira ${ }^{1}$; Marcelo Alves Pinto ${ }^{1}$ ${ }^{1}$ Fiocruz/IOC;

${ }^{2}$ UFF - Universidade Federal Fluminense;

${ }^{3}$ ANVISA.

Introduction: Hepatitis $\mathrm{C}$ is a disease with a global prevalence of $3 \%$ of cases of hepatitis $\mathrm{C}$ virus (HCV) infection, which corresponds to 130 million infected people worldwide. Recently, new drugs have emerged for the treatment of HCV infection with fewer side effects and greater efficacy, composing direct action antivirals (DAA) regimens. However, the search for new antiviral agents continues to be necessary due to HCV Infections non-Responsible to them.

Objective: In this work, we applied a new "in vitro" screening methodology to access a possible antiviral activity from naturals or chemicals compounds.

Methodology: Molecules: we tested 360 naturals and chemicals compounds. Cells: Huh 7.0 (human hepatocarcinoma cell line). Ethics Committee Approval: This work was approved by a National Ethical Council for Research (CONEP): 83368818.1.0000.5248.Production of HCVcc: Huh 7.0 cells were infected with HCV positive human serum containing a viral load of 105 copies / $\mathrm{mL}$ and maintained in culture with DMEM medium without fetal serum for 5 days. NS3 activity assay: we performed the evaluation of NS3 activity from virions produced during the treatments, using a kit Anaspec (Fremont, California, United States). RT-qPCR: followed by the RT-qPCR kit for quantification of HCV RNA (Bioclin, Belo Horizonte, Minas Gerais, Brasil).ELISA technique: We assessed ELISA Kits PeproTech (Rocky Hill, New Jersey, United States, USA). Biochemical analysis: We quantified the AST, ALT, Albumin and LDH using kits from Doles (Goiania, GO, Brazil). Data analysis: Representative results from 3 independent experiments. Statistical tests used were described in figure legends. $P$ values of 0.05 or less were considered significant.

Results: we tested 360 compounds and discovered the promising inhibitory action of two compounds (T-MXI and T-DAG) in the range of [100 $\mu \mathrm{g} / \mathrm{mL}]$, on the NS3 viral protease, with an inhibitory profile of $90 \%$ and $80 \%$ respectively, and Simeprevir (NS3 inhibitor used in the clinic) inhibited $70 \%$. We also tested the cytotoxicity by quantifying LDH after 24 hours of treatment in Huh 7.0 cells and no one showed cytotoxicity. We also performed RT-qPCR after 7 days of incubation within the treatment, and a decrease of approximately $60 \%$ and $75 \%$, in relation to the not treated control, was observed. we noted that Simeprevir inhibited 50\%.To access the immune response from treatments, we performed the ELISA and observed that both compounds were able to increase the release of pro inflammatory cytokines about $50 \% \sim 60 \%$, stimulating an antiviral response. We also evaluated the hepatic enzymes release in infections treatments, which lowered about $80 \%$ and $60 \%$ respectively. finally, we assessed a microcytopathic effect through LDH released, demonstrated an inhibition of $70 \%$ and $55 \%$ by both.

Conclusion: Our results may contribute for the discovery of new antiviral drugs for treatment of $\mathrm{HCV}$ infection cases that are not responsible to the currently available DAA.

Keywords: Protease; HCV; Screening 